\title{
A Cohomological Proof that Real Representations of Semisimple Lie Algebras Have $\mathbb{Q}$-Forms
}

Dave Witte MORRIS

Department of Mathematics and Computer Science, University of Lethbridge, Lethbridge, Alberta, T1K 3M4, Canada

E-mail: Dave.Morris@uleth.ca

URL: http://people.uleth.ca/ dave.morris/

Received October 17, 2014, in final form April 14, 2015; Published online April 27, 2015

http://dx.doi.org/10.3842/SIGMA.2015.034

\begin{abstract}
A Lie algebra $\mathfrak{g}_{\mathbb{Q}}$ over $\mathbb{Q}$ is said to be $\mathbb{R}$-universal if every homomorphism from $\mathfrak{g}_{\mathbb{Q}}$ to $\mathfrak{g l}(n, \mathbb{R})$ is conjugate to a homomorphism into $\mathfrak{g l}(n, \mathbb{Q})$ (for every $n$ ). By using Galois cohomology, we provide a short proof of the known fact that every real semisimple Lie algebra has an $\mathbb{R}$-universal $\mathbb{Q}$-form. We also provide a classification of the $\mathbb{R}$-universal Lie algebras that are semisimple.
\end{abstract}

Key words: semisimple Lie algebra; finite-dimensional representation; global field; Galois cohomology; linear algebraic group; Tits algebra

2010 Mathematics Subject Classification: 17B10; 17B20; 11E72; 20G30

\section{Introduction}

Definition 1.1. Let $\mathfrak{g}_{\mathbb{Q}}$ be a Lie algebra over $\mathbb{Q}$. (All Lie algebras and all representations are assumed to be finite-dimensional.)

1. $\mathfrak{g}_{\mathbb{Q}}$ is universal for real representations (or $\mathbb{R}$-universal, for short) if every real representation of $\mathfrak{g}_{\mathbb{Q}}$ has a $\mathbb{Q}$-form [6, Definition 7.1]. This means that if $\rho: \mathfrak{g}_{\mathbb{Q}} \rightarrow \mathfrak{g l}(n, \mathbb{R})$ is any $(\mathbb{Q}$-linear) Lie algebra homomorphism, then there exists $M \in \mathrm{GL}(n, \mathbb{R})$, such that $M \rho(x) M^{-1} \in \mathfrak{g l}(n, \mathbb{Q})$, for every $x \in \mathfrak{g}_{\mathbb{Q}}$.

2. $\mathfrak{g}_{\mathbb{Q}}$ is a $\mathbb{Q}$-form of a real Lie algebra $\mathfrak{g}_{\mathbb{R}}$ if $\mathfrak{g}_{\mathbb{Q}} \otimes_{\mathbb{Q}} \mathbb{R} \cong \mathfrak{g}_{\mathbb{R}}$.

This note uses Galois cohomology to present a short proof of the following known result, which was first obtained by M.S. Raghunathan $[9, \S 3]$ in the important special case where $\mathfrak{g}_{\mathbb{R}}$ is compact.

Proposition 1.2 ([6, Theorem 1.2]). Every real semisimple Lie algebra has a $\mathbb{Q}$-form that is $\mathbb{R}$-universal.

The proof in [6] constructs an $\mathbb{R}$-universal $\mathbb{Q}$-form explicitly, and is rather tedious, but a much nicer proof was given by G. Prasad and A. Rapinchuk [8, Proposition 3 and Remark 3]. Assuming some fundamental results of J. Tits [11], our proof in Section 2 is a bit shorter and more direct. (On the other hand, we provide less information about the $\mathbb{Q}$-form than is supplied in [8].)

Section 3 gives an explicit characterization of the $\mathbb{R}$-universal Lie algebras that are absolutely simple over $\mathbb{Q}$, and, for completeness, Section 4 explains how to extend this to the class of all semisimple Lie algebras.

Due to the well-known correspondence between $\mathbb{Q}$-forms and arithmetic subgroups, Proposition 1.2 has the following consequence in the theory of discrete subgroups: 
Corollary 1.3. Let $G$ be a connected, semisimple Lie group with finite center. Then there is a discrete subgroup $\Gamma$ of $G$, such that

(1) $G / \Gamma$ has finite volume (so $\Gamma$ is a "lattice" in $G$ ), and

(2) if $\rho: G \rightarrow \mathrm{GL}(n, \mathbb{R})$ is any finite-dimensional representation of $G$, then $\rho(\Gamma)$ is conjugate to a subgroup of $\mathrm{GL}(n, \mathbb{Z})$.

\section{Proof of the results stated in the introduction}

We begin by recalling a result of J. Tits that uses Galois cohomology to characterize the irreducible representations of semisimple algebraic groups over fields that are not algebraically closed. However, we will state this work in the setting of Lie algebras, rather than algebraic groups. (We deal only with semisimple Lie algebras and semisimple groups, and the fields under consideration in this paper are all of characteristic zero, so no difficulties arise in making this translation [1, Proposition 7.3.1(iii), p. 393].)

Definition 2.1 ([11, $\S 4.2])$. Suppose $\mathfrak{g}$ is a semisimple Lie algebra over a subfield $F$ of $\mathbb{C}$, and let $\mathbf{G}$ be the corresponding simply connected, semisimple algebraic group over $F$. It is well known that there is a (unique) quasi-split, simply connected algebraic group $\mathbf{G}^{q}$ over $F$, and a 1cocycle $\xi: \operatorname{Gal}(\bar{F} / F) \rightarrow \overline{\mathbf{G}}^{q}$, where $\overline{\mathbf{G}}^{q}$ is the adjoint group of $\mathbf{G}^{q}$, such that $\mathbf{G}$ is $F$-isomorphic to the Galois twist ${ }^{\xi} \mathbf{G}^{q}$. This cocycle determines a cohomology class $[\xi] \in H^{1}\left(F ; \overline{\mathbf{G}}^{q}\right)$.

Letting $Z\left(\mathbf{G}^{q}\right)$ be the center of $\mathbf{G}^{q}$, the short exact sequence $e \rightarrow Z\left(\mathbf{G}^{q}\right) \rightarrow \mathbf{G}^{q} \rightarrow \overline{\mathbf{G}}^{q} \rightarrow e$ yields a corresponding long exact sequence of Galois cohomology sets, including a connecting map $\delta_{*}: H^{1}\left(F ; \overline{\mathbf{G}}^{q}\right) \rightarrow H^{2}\left(F ; Z\left(\mathbf{G}^{q}\right)\right)$. Hence, we have a cohomology class $\delta_{*}[\xi] \in H^{2}\left(F ; Z\left(\mathbf{G}^{q}\right)\right)$. (If we took a bit more care to ensure that it is well-defined, this would be the Tits class of $\mathbf{G}[4$, p. 426].)

Now, fix a maximal $F$-torus $\mathbf{T}$ of $\mathbf{G}^{q}$ that contains a maximal $F$-split torus, and suppose $\lambda$ is a weight of $\mathbf{T}$ that is invariant under the *-action of the Galois group $\operatorname{Gal}(\bar{F} / F)$. Then the restriction of $\lambda$ to $Z\left(\mathbf{G}^{q}\right)$ is a $\operatorname{Gal}(\bar{F} / F)$-equivariant homomorphism from $Z\left(\mathbf{G}^{q}\right)$ to the group $\boldsymbol{\mu}$ of roots of unity in $\mathbb{C}$, so it induces a homomorphism $\lambda_{*}: H^{2}\left(F ; Z\left(\mathbf{G}^{q}\right)\right) \rightarrow H^{2}(F ; \boldsymbol{\mu})$. Therefore, we may define

$$
\beta_{\mathfrak{g}, F}(\lambda)=\lambda_{*} \delta_{*}[\xi] \in H^{2}(F ; \boldsymbol{\mu}) .
$$

Remark 2.2. For a semisimple group $\mathbf{G}$ that is defined over a field $F$, the definition of the *-action of $\operatorname{Gal}(\bar{F} / F)$ on the weights of a maximal torus $\mathbf{T}$ presupposes that $\mathbf{T}$ is defined over $F$ and contains a maximal $F$-split torus of $\mathbf{G}[7$, p. 66]. When $\mathbf{G}$ is defined over $\mathbb{Q}$, we will use the *-actions of both $\operatorname{Gal}(\overline{\mathbb{Q}} / \mathbb{Q})$ and $\operatorname{Gal}(\mathbb{C} / \mathbb{R})$, so we will assume that $\mathbf{T}$ is defined over $\mathbb{Q}$ (so it is also defined over $\mathbb{R}$ ) and contains both a maximal $\mathbb{Q}$-split torus and a maximal $\mathbb{R}$-split torus. To see that such a choice of $\mathbf{T}$ is always possible, let $\mathbf{T}_{1}$ be any maximal $\mathbb{Q}$-split torus of $\mathbf{G}$. Then there is a maximal $\mathbb{Q}$-torus $\mathbf{T}$ of the centralizer $\mathbf{C}_{\mathbf{G}}\left(\mathbf{T}_{1}\right)$ that contains a maximal $\mathbb{R}$-split torus [7, Corollary 2 of Proposition 7.8, p. 418], and it is clear that $\mathbf{T}$ has the desired properties.

Proposition 2.3 ([11, Theorem 7.2 and Lemma 7.4]). Suppose $\mathfrak{g}$ is a semisimple Lie algebra over a subfield $F$ of $\mathbb{C}$, and $\lambda$ is a dominant weight. Then:

1. There is an irreducible representation ${ }^{F} \rho_{\lambda}: \mathfrak{g} \rightarrow \mathfrak{g l}(n, F)$, for some $n$, such that ${ }^{F} \rho_{\lambda} \otimes_{F} \mathbb{C}$ has an irreducible summand with highest weight $\lambda$. Furthermore, ${ }^{F} \rho_{\lambda}$ is unique up to isomorphism.

2. ${ }^{F} \rho_{\lambda_{1}} \cong{ }^{F} \rho_{\lambda_{2}}$ if and only if $\lambda_{1}$ and $\lambda_{2}$ are in the same orbit of the $*$-action of $\operatorname{Gal}(\bar{F} / F)$.

3. ${ }^{F} \rho_{\lambda} \otimes_{F} \mathbb{C}$ is irreducible if and only if: 
(a) $\lambda$ is invariant under the $*$-action of $\mathrm{Gal}(\bar{F} / F)$, and

(b) $\beta_{\mathfrak{g}, F}(\lambda)$ is the trivial element of $H^{2}(F ; \boldsymbol{\mu})$.

Furthermore, every $F$-irreducible representation of $\mathfrak{g}$ is isomorphic to ${ }^{F} \rho_{\lambda}$, for some dominant weight $\lambda$.

Corollary 2.4. Suppose $\mathfrak{g}$ is a semisimple Lie algebra over $\mathbb{Q}$, such that $\mathfrak{g}$ splits over a quadratic extension of $\mathbb{Q}$, and, for every dominant weight $\lambda$ of $\mathbf{T}$ :

if $\lambda$ is invariant under the $*$-action of $\operatorname{Gal}(\mathbb{C} / \mathbb{R})$, and $\beta_{\mathfrak{g}, \mathbb{R}}(\lambda)=0$,

then $\lambda$ is also invariant under the $*$-action of $\operatorname{Gal}(\overline{\mathbb{Q}} / \mathbb{Q})$, and $\beta_{\mathfrak{g}, \mathbb{Q}}(\lambda)=0$.

Then $\mathfrak{g}$ is $\mathbb{R}$-universal.

Proof. Since representations of $\mathfrak{g}$ are completely reducible, it suffices to show that every irreducible real representation of $\mathfrak{g}$ has a $\mathbb{Q}$-form. Specifically, we will show that ${ }^{\mathbb{Q}} \rho_{\lambda}$ is a $\mathbb{Q}$-form of ${ }^{\mathbb{R}} \rho_{\lambda}$, for every dominant weight $\lambda$.

We begin by showing that if ${ }^{\mathbb{R}} \rho_{\lambda} \otimes_{\mathbb{R}} \mathbb{C}$ is irreducible, then ${ }^{\mathbb{Q}} \rho_{\lambda} \otimes_{\mathbb{Q}} \mathbb{C}$ is irreducible. From Proposition 2.3(3) (with $F=\mathbb{R}$ ), we know that $\lambda$ is invariant under the $*$-action of $\mathrm{Gal}(\mathbb{C} / \mathbb{R})$, and that $\beta_{\mathfrak{g}, \mathbb{R}}(\lambda)=0$. By assumption, this implies that $\lambda$ is invariant under the $*$-action of $\operatorname{Gal}(\overline{\mathbb{Q}} / \mathbb{Q})$, and that $\beta_{\mathfrak{g}, \mathbb{Q}}(\lambda)=0$. Then, from Proposition 2.3(3) (with $F=\mathbb{Q}$ ), we conclude that ${ }^{\mathbb{Q}} \rho_{\lambda} \otimes \mathbb{Q} \mathbb{C}$ is irreducible, as desired.

Since $\mathfrak{g}_{\mathbb{Q}}$ splits over a quadratic extension, we know that ${ }^{\mathbb{Q}} \rho_{\lambda} \otimes_{\mathbb{Q}} \mathbb{C}$ is either irreducible or the direct sum of two irreducibles $\left[6\right.$, Corollary 3.2(2)]. Therefore, ${ }^{\mathbb{Q}} \rho_{\lambda} \otimes_{\mathbb{Q}} \mathbb{C}$ and ${ }^{\mathbb{R}} \rho_{\lambda} \otimes_{\mathbb{R}} \mathbb{C}$ have the same number of irreducible constituents. (Namely, either they are both irreducible, or they are both the direct sum of 2 irreducibles.) Since ${ }^{\mathbb{R}} \rho_{\lambda}$ is a summand of ${ }^{\mathbb{Q}} \rho_{\lambda} \otimes \mathbb{Q} \mathbb{R}$, this implies that ${ }^{\mathbb{R}} \rho_{\lambda} \cong \mathbb{Q}_{\rho_{\lambda} \otimes \mathbb{Q}} \mathbb{R}$, so ${ }^{\mathbb{Q}} \rho_{\lambda}$ is a $\mathbb{Q}$-form of ${ }^{\mathbb{R}} \rho_{\lambda}$.

We will also use the following variant of a basic fact in the theory of Galois cohomology:

Lemma 2.5 ([3, Theorem $5.1 \mathrm{~b}$, p. 77]). If $\mathbf{G}$ is a connected, semisimple algebraic group over $\mathbb{Q}$, and $L$ is any imaginary quadratic extension of $\mathbb{Q}$, then the natural restriction map $H^{1}(L / F ; \mathbf{G}(L)) \rightarrow H^{1}(\mathbb{R} ; \mathbf{G})$ is surjective.

Proof. (The author thanks A. Rapinchuk for suggesting this argument.) Let $\sigma$ be the nontrivial element of $\operatorname{Gal}(\mathbb{C} / \mathbb{R})$. It is well known that any cohomology class in $H^{1}(\mathbb{R} ; \mathbf{G})$ is represented by an element $t$ of a maximal torus $\mathbf{T}(\mathbb{C})$ of $\mathbf{G}(\mathbb{C})$, such that $t t^{\sigma}=1$, and, since the variety of maximal tori has weak approximation, that $\mathbf{T}$ may be chosen to be defined over $\mathbb{Q}$ (cf. proof of [7, Proposition 6.17, p. 337]).

Let $\mathbf{R}=\operatorname{Res}_{L / \mathbb{Q}} \mathbf{T}$ be the torus obtained from $\mathbf{T}$ by restriction of scalars from $L$ to $\mathbb{Q}$. Then there is a natural isomorphism $\varphi: \mathbf{T}(\mathbb{C}) \rightarrow \mathbf{R}(\mathbb{R})$, such that $\varphi(\mathbf{T}(L))=\mathbf{R}(\mathbb{Q})$, and there is a $\mathbb{Q}$-automorphism $\tau$ of $\mathbf{R}$, such that $\varphi\left(x^{\sigma}\right)=\varphi(x)^{\tau}$, for all $x \in \mathbf{T}(\mathbb{C})$.

Let $\mathbf{R}^{(1)}=\left\{r \in \mathbf{R} \mid r r^{\tau}=1\right\}$. We claim that this is a subtorus of $\mathbf{R}$. Since $\mathbf{T}$ is defined over $\mathbb{Q}$, there is an $L$-isomorphism $\psi: \mathbf{R} \rightarrow \mathbf{T} \times \mathbf{T}$, such that $\psi(\mathbf{R}(\mathbb{R}))=\left\{\left(x, x^{\sigma}\right) \mid x \in \mathbf{T}(\mathbb{C})\right\}$. Therefore, if we define $(x, y)^{\pi}=(y, x)$, then $\psi\left(r^{\tau}\right)=\psi(r)^{\pi}$ for all $r \in \mathbf{R}$. So $\psi\left(\mathbf{R}^{(1)}\right)=$ $\left\{\left(x, x^{-1}\right) \mid x \in \mathbf{T}\right\}$ is a subtorus of $\mathbf{T} \times \mathbf{T}$, as claimed.

Now, $\mathbf{R}^{(1)}$ is a torus that is defined over $\mathbb{Q}$ (since the automorphism $\tau$ is defined over $\mathbb{Q}$ ), and all $\mathbb{Q}$-tori have weak approximation at the infinite place [7, Corollary 1 of Proposition 7.8, p. 418], so $\mathbf{R}^{(1)}(\mathbb{Q})$ is dense in $\mathbf{R}^{(1)}(\mathbb{R})$. Hence, some $q \in \mathbf{R}^{(1)}(\mathbb{Q})$ is in the same connected component of $\mathbf{R}^{(1)}(\mathbb{R})$ as $\varphi(t)$. Then, letting $q^{\prime}=\varphi^{-1}(q)$, we see that $q^{\prime}$ is in the same connected component of $\left\{w \in \mathbf{T}(\mathbb{R}) \mid w w^{\sigma}=1\right\}$ as $t$, so $q^{\prime}$ represents the same cohomology class as $t$ in $H^{1}(\mathbb{R} ; \mathbf{G})$. However, since $q^{\prime} \in \mathbf{T}(L)$, we see that the cohomology class of $q^{\prime}$ is in the image of $H^{1}(L / F ; \mathbf{G}(L))$, as desired. 
Proof of Proposition 1.2. Suppose $\mathfrak{g}_{\mathbb{R}}$ is a real semisimple Lie algebra, and let $\mathbf{G}$ be the simply connected, semisimple $\mathbb{R}$-algebraic group whose Lie algebra is $\mathfrak{g}_{\mathbb{R}}$. As in Definition 2.1, write $\mathbf{G}={ }^{\xi} \mathbf{G}^{q}$, where $\xi: \operatorname{Gal}(\mathbb{C} / \mathbb{R}) \rightarrow \overline{\mathbf{G}}^{q}$ is a 1-cocycle and $\mathbf{G}^{q}$ is quasi-split. Let $L=\mathbb{Q}[i]$. By choosing an appropriate $\mathbb{Q}$-form, we may assume that $\mathbf{G}^{q}$ is a quasi-split $\mathbb{Q}$-group that splits over $L$, and that the $*$-action of $\operatorname{Gal}(L / \mathbb{Q})$ is the same as the $*$-action of $\operatorname{Gal}(\mathbb{C} / \mathbb{R})$.

Let $\sigma$ be the nontrivial element of $\operatorname{Gal}(\mathbb{C} / \mathbb{R})$, fix a representative $a \in \mathbf{G}^{q}(\mathbb{C})$ of $\xi(\sigma) \in \overline{\mathbf{G}}^{q}(\mathbb{C})$, and let $z=a^{\sigma} a$. Since $\sigma^{2}$ is trivial and $\xi$ is a 1-cocycle, we know that $z$ is trivial in $\overline{\mathbf{G}}^{q}$, so $z \in Z\left(\mathbf{G}^{q}\right)(\mathbb{C})$. This implies that $a$ commutes with ${ }^{\sigma} a$, so $\sigma$ fixes $z$, which means $z \in Z\left(\mathbf{G}^{q}\right)(\mathbb{R})$.

Let $\mathbf{H}$ be the product of the almost simple factors of $\mathbf{G}^{q}$ that are absolutely almost simple and of type ${ }^{2} A_{n}$ (more concretely, $\mathbf{H}$ is the product of the factors that are isomorphic to $\mathbf{S U}(k, \ell)$, for some $k$ and $\ell$ ), let $Z(\mathbf{H})^{2}=\left\{w^{2} \mid w \in Z(\mathbf{H})(\mathbb{C})\right\}$, let $\underline{\mathbf{G}}^{q}=\mathbf{G}^{q} / Z(\mathbf{H})^{2}$, and let $\underline{z}$ be the image of $z$ in $\underline{\mathbf{G}}^{q}$. Note that $\underline{\mathbf{G}}^{q}$ is a $\mathbb{Q}$-group (since $Z(\mathbf{H})^{2}$ is a $\mathbb{Q}$-subgroup of $\mathbf{G}^{q}$ ).

We claim that we may assume $\underline{z} \in Z\left(\underline{\mathbf{G}}^{q}\right)(\mathbb{Q})$. While proving this, we may consider each simple factor individually, so there is no harm in assuming $\mathbf{G}^{q}$ is almost simple. This allows us to furthermore assume that $\underline{\mathbf{G}}^{q}$ is absolutely almost simple. (Otherwise, since every $\mathbb{C}$-group is split, we could assume $\xi$ is trivial.) Also, since $|\operatorname{Gal}(\mathbb{C} / \mathbb{R})|=2$, we may assume, by replacing $a$ with $a w$ for an appropriately chosen $w \in\langle z\rangle$, that $|\underline{z}|$ is a power of 2 . Assuming, as we may, that $\underline{z}$ is nontrivial, this implies that $\mathbf{G}^{q}$ is not of type ${ }^{1,2} E_{6}$. Then $Z\left(\underline{\mathbf{G}}^{q}\right)(\mathbb{R})=Z\left(\underline{\mathbf{G}}^{q}\right)(\mathbb{Q})$. (If $\mathbf{G}^{q}$ is of type ${ }^{2} A_{n}$, then the definition of $\underline{\mathbf{G}}^{q}$ implies $\left|Z\left(\underline{\mathbf{G}}^{q}\right)\right| \leq 2$, so every element of $Z\left(\underline{\mathbf{G}}^{q}\right)$ is defined over $\mathbb{Q}$. If $\mathbf{G}^{q}$ is not of this type, then the desired conclusion can be verified by noting that $Z\left(\mathbf{G}^{q}\right)$ is either $\boldsymbol{\mu}_{n}, \boldsymbol{\mu}_{2} \times \boldsymbol{\mu}_{2}, \mathbf{R}_{L / \mathbb{Q}} \boldsymbol{\mu}_{2}, \mathbf{R}_{L / \mathbb{Q}}^{(1)} \boldsymbol{\mu}_{2}$, or $\mathbf{R}_{L / \mathbb{Q}}^{(1)} \boldsymbol{\mu}_{4}$ [7, p. 332].) This completes the proof of the claim.

The claim of the preceding paragraph implies that the cyclic subgroup $\langle\underline{z}\rangle$ generated by $\underline{z}$ is defined over $\mathbb{Q}$. Hence, the quotient $\widetilde{\mathbf{G}}^{q}=\mathbf{G}^{q} /\left\langle z, Z(\mathbf{H})^{2}\right\rangle$ is a semisimple $\mathbb{Q}$-group. Now, since $a^{\sigma} a=z$ is trivial in $\widetilde{\mathbf{G}}^{q}$, we know that $\xi$ lifts to a 1 -cocycle $\widetilde{\xi}: \operatorname{Gal}(\mathbb{C} / \mathbb{R}) \rightarrow \widetilde{\mathbf{G}}^{q}$. Then Lemma 2.5 implies that, after replacing $\widetilde{\xi}$ with a cohomologous cocycle, we may assume $\widetilde{\xi}$ is the restriction of a 1-cocycle $\zeta: \operatorname{Gal}(L / \mathbb{Q}) \rightarrow \widetilde{\mathbf{G}}^{q}(L)$. Let $\mathbf{G}_{\mathbb{Q}}=\zeta \mathbf{G}^{q}$, so $\mathbf{G}_{\mathbb{Q}}$ is a $\mathbb{Q}$-group that is $L$-split, and is isomorphic to $\mathbf{G}$ over $\mathbb{R}$. Also, let $\mathfrak{g}$ be the Lie algebra of $\mathbf{G}_{\mathbb{Q}}$.

To complete the proof, we show that $\mathfrak{g}$ is $\mathbb{R}$-universal, by verifying (2.1). To this end, let $\lambda$ be a $\operatorname{Gal}(\mathbb{C} / \mathbb{R})$-invariant dominant weight, such that $\beta_{\mathfrak{g}, \mathbb{R}}(\lambda)=0$. Since $\mathbf{G}^{q}$ is $L$-split and the *-action of $\operatorname{Gal}(L / \mathbb{Q})$ is the same as the $*$-action of $\operatorname{Gal}(\mathbb{C} / \mathbb{R})$ (by the choice of the $\mathbb{Q}$-form of $\left.\mathbf{G}^{q}\right)$, we know that $\lambda$ is invariant under $\operatorname{Gal}(\overline{\mathbb{Q}} / \mathbb{Q})$.

Since $\zeta$ is a 1-cocycle into $\widetilde{\mathbf{G}}^{q}=\mathbf{G}^{q} /\left\langle z, Z(\mathbf{H})^{2}\right\rangle$, we know that, in the notation of Definition 2.1 with $F=\mathbb{Q}$, we have $\delta_{*}[\zeta] \in H^{2}\left(\mathbb{Q} ;\left\langle z, Z(\mathbf{H})^{2}\right\rangle\right)$. Therefore, in order to show that $\beta_{\mathfrak{g}, \mathbb{Q}}(\lambda)=$ $\lambda_{*} \delta_{*}[\zeta]=0$, it suffices to show that $\lambda$ is trivial on both $z$ and $Z(\mathbf{H})^{2}$. Note that, in the notation of Definition 2.1 with $F=\mathbb{R}$, we have $\lambda_{*} \delta_{*}[\xi]=\beta_{\mathfrak{g}, \mathbb{R}}(\lambda)=0$. Under the natural identification of $H^{2}\left(\mathbb{R} ; Z\left(\mathbf{G}^{q}\right)\right)$ with

$$
\left\{w \in Z\left(\mathbf{G}^{q}\right) \mid{ }^{\sigma} w=w\right\} /\left\{w^{\sigma} w \mid w \in Z\left(\mathbf{G}^{q}\right)\right\},
$$

we have $\delta_{*}[\xi]=[z]$, so this means $\lambda(z)=1$ (since $\omega \bar{\omega}=1$ for all $\omega \in \boldsymbol{\mu}$ ). Furthermore, since the restriction of $\lambda$ to $Z\left(\mathbf{G}^{q}\right)$ is a $\operatorname{Gal}(\mathbb{C} / \mathbb{R})$-equivariant homomorphism, and $Z(\mathbf{H})(\mathbb{R})=Z(\mathbf{H})$ (cf. [7, p. 332]), we have $\lambda(Z(\mathbf{H})) \subseteq \boldsymbol{\mu}(\mathbb{R})=\{ \pm 1\}$, so $\lambda$ is also trivial on $Z(\mathbf{H})^{2}$.

Proof of Corollary 1.3. Choose an $\mathbb{R}$-universal $\mathbb{Q}$-form $\mathfrak{g}_{\mathbb{Q}}$ of the Lie algebra $\mathfrak{g}$ of $G$, let $\overline{\mathbf{G}}$ be the corresponding adjoint $\mathbb{Q}$-group, and let $\Gamma=\{g \in G \mid \operatorname{Ad} g \in \overline{\mathbf{G}}(\mathbb{Z})\}$, so $\Gamma$ is a lattice in $G$ (cf. [7, Theorem 4.14, p. 220]).

Suppose $\rho: G \rightarrow \operatorname{GL}(n, \mathbb{R})$ is a finite-dimensional representation of $G$. By replacing $\rho$ with a conjugate, we may assume $d \rho\left(\mathfrak{g}_{\mathbb{Q}}\right) \subseteq \mathfrak{g l}(n, \mathbb{Q})$ (because $\mathfrak{g}_{\mathbb{Q}}$ is $\mathbb{R}$-universal). Then the corresponding representation $\widetilde{\rho}$ of the universal cover $\mathbf{G}$ of $\overline{\mathbf{G}}$ is defined over $\mathbb{Q}$, so there is a $\widetilde{\rho}(\mathbf{G}(\mathbb{Z}))$ invariant $\mathbb{Z}$-lattice in $\mathbb{Q}^{n}[7$, Remark on p. 173]. Then, since $\widetilde{\rho}(\mathbf{G}(\mathbb{Z}))$ contains a finite-index 
subgroup of $\rho(\Gamma)$, there is also a $\rho(\Gamma)$-invariant $\mathbb{Z}$-lattice in $\mathbb{Q}^{n}$, so $\rho(\Gamma)$ is conjugate to a subgroup of $\operatorname{GL}(n, \mathbb{Z})$.

\section{$3 \quad \mathbb{R}$-universal absolutely simple Lie algebras}

This section provides a classification of the absolutely simple Lie algebras over $\mathbb{Q}$ that are $\mathbb{R}$ universal. (To say $\mathfrak{g}_{\mathbb{Q}}$ is absolutely simple means that $\mathfrak{g}_{\mathbb{Q}} \otimes_{\mathbb{Q}} \mathbb{C}$ is simple. See Section 4 for a discussion of $\mathbb{R}$-universal Lie algebras that do not have this property.) We record a few observations before proceeding with case-by-case analysis.

Notation 3.1. We assume $\mathfrak{g}$ is a semisimple Lie algebra over a field $F$ of characteristic 0 , that $\lambda$ is a dominant weight, and the other notation of Definition 2.1. Furthermore, we let $Z^{*}$ be the finite, abelian group of all homomorphisms from $Z\left(\mathbf{G}^{q}\right)$ to $\boldsymbol{\mu}$.

Proposition 3.2 ([11, Corollary 3.5, $\S 4.2$, and Lemma 7.4]). Let $L$ be the center of the algebra $D_{\mathfrak{g}, F}(\lambda):=\operatorname{End}_{\mathfrak{g}}\left({ }^{F} \rho_{\lambda}\right)$ (which, by Schur's lemma, is a division algebra). Then:

(1) $\operatorname{Gal}(\bar{F} / L)=\{\sigma \in \operatorname{Gal}(\bar{F} / F) \mid \sigma(\lambda)=\lambda\}$ (for the $*$-action of $\operatorname{Gal}(\bar{F} / F)$ ), and

(2) $\beta_{\mathfrak{g}, L}(\lambda)=\left[D_{\mathfrak{g}, F}(\lambda)\right]$, after identifying $H^{2}(L ; \boldsymbol{\mu})$ with the Brauer group of $L$.

Definition 3.3. We will call $D_{\mathfrak{g}, F}(\lambda)$ the Tits algebra of $\mathfrak{g}$ corresponding to the weight $\lambda$ over $F$. (However, this name is used in the literature for a slightly different algebra that is Brauer equivalent to $D_{\mathfrak{g}, F}(\lambda)[4, \S 27 \mathrm{~A}$, p. 377].)

Lemma 3.4. If $\mathfrak{g}$ is a semisimple Lie algebra over $\mathbb{Q}$, then the following are equivalent:

1. $\mathfrak{g}$ is $\mathbb{R}$-universal.

2. ${ }^{\mathbb{Q}} \rho_{\lambda} \otimes \mathbb{Q} \mathbb{R} \cong \mathbb{R} \rho_{\lambda}$, for every dominant weight $\lambda$.

3. $D_{\mathfrak{g}, \mathbb{Q}}(\lambda) \otimes_{\mathbb{Q}} \mathbb{R}$ is a division algebra, for every dominant weight $\lambda$.

4. $D_{\mathfrak{g}, \mathbb{Q}}(\lambda) \otimes_{\mathbb{Q}} \mathbb{R} \cong D_{\mathfrak{g}, \mathbb{R}}(\lambda)$, for every dominant weight $\lambda$.

Proof. $(1 \Rightarrow 2)$ Let $\rho$ be a $\mathbb{Q}$-form of ${ }^{\mathbb{R}} \rho_{\lambda}$. Then $\rho$ must be $\mathbb{Q}$-irreducible (since $\rho \otimes_{\mathbb{Q}} \mathbb{R} \cong \mathbb{R} \rho_{\lambda}$ is $\mathbb{R}$-irreducible) and

$$
\rho \otimes_{\mathbb{Q}} \mathbb{C} \cong\left(\rho \otimes_{\mathbb{Q}} \mathbb{R}\right) \otimes_{\mathbb{R}} \mathbb{C} \cong{ }^{\mathbb{R}} \rho_{\lambda} \otimes_{\mathbb{R}} \mathbb{C}
$$

has an irreducible summand with highest weight $\lambda$. Therefore $\rho \cong{ }^{\mathbb{Q}} \rho_{\lambda}$.

$(2 \Rightarrow 1){ }^{\mathbb{Q}} \rho_{\lambda}$ is a $\mathbb{Q}$-form of ${ }^{\mathbb{R}} \rho_{\lambda}$.

$(2 \Leftrightarrow 3 \Leftrightarrow 4)$ We have $\operatorname{End}_{\mathfrak{g}}\left({ }^{\mathbb{Q}} \rho_{\lambda} \otimes_{\mathbb{Q}} \mathbb{R}\right)=D_{\mathfrak{g}, \mathbb{Q}}(\lambda) \otimes_{\mathbb{Q}} \mathbb{R}$. So ${ }^{\mathbb{Q}} \rho_{\lambda} \otimes_{\mathbb{Q}} \mathbb{R}$ is irreducible (and hence equal to ${ }^{\mathbb{R}} \rho_{\lambda}$ ) if and only if $D_{\mathfrak{g}, \mathbb{Q}}(\lambda) \otimes_{\mathbb{Q}} \mathbb{R}$ is a division algebra (and hence equal to $D_{\mathfrak{g}, \mathbb{R}}(\lambda)$ ).

Remark 3.5. It is immediate from Definition 2.1 that if the $*$-invariant weights $\lambda_{1}$ and $\lambda_{2}$ have the same restriction to $Z\left(\mathbf{G}^{q}\right)$ (that is, if they represent the same element of $Z^{*}$ ), then $\beta_{\mathfrak{g}, F}\left(\lambda_{1}\right)=\beta_{\mathfrak{g}, F}\left(\lambda_{2}\right)$. Therefore, if $\lambda$ is a dominant weight in the root lattice (so $\lambda$ is trivial on $\left.Z\left(\mathbf{G}^{q}\right)\right)$, and $\lambda$ is invariant under the $*$-action of $\operatorname{Gal}(\bar{F} / F)$, then $D_{\mathfrak{g}, F}(\lambda)=F$.

Lemma 3.6. Let $\mathfrak{g}$ be a semisimple Lie algebra over $\mathbb{Q}$. If $\mathfrak{g}$ is $\mathbb{R}$-universal, then $\mathfrak{g}$ is inner over some imaginary quadratic extension $L$ of $\mathbb{Q}$, and the $*$-action of $\operatorname{Gal}(L / \mathbb{Q})$ is the same as the *-action of $\operatorname{Gal}(\mathbb{C} / \mathbb{R})$. 
Proof. Assume $\mathfrak{g}$ is not inner, for otherwise the desired conclusions are obvious. Let $L$ be the (unique) minimal extension of $\mathbb{Q}$ over which $\mathfrak{g}$ becomes inner, and choose some dominant weight $\lambda$ that is not fixed by any nontrivial element of $\operatorname{Gal}(L / \mathbb{Q})$. We know that $L$ is the center of $D_{\mathfrak{g}, \mathbb{Q}}(\lambda)$ (see Proposition 3.2(1)) and that $D_{\mathfrak{g}, \mathbb{Q}}(\lambda) \otimes_{\mathbb{Q}} \mathbb{R}$ is a division algebra (see Lemma 3.4(3)). Since $L$ is not $\mathbb{Q}$, this implies it is an imaginary quadratic extension of $\mathbb{Q}$.

It now suffices to show that every every $\operatorname{Gal}(\mathbb{C} / \mathbb{R})$-invariant dominant weight $\lambda$ is also $\operatorname{Gal}(L / \mathbb{Q})$-invariant. Suppose not. By replacing $\lambda$ with an appropriate positive integer multiple, we may assume it is in the root lattice, so $D_{\mathfrak{g}, \mathbb{R}}(\lambda)=\mathbb{R}$ (see Remark 3.5). Therefore, we must have $D_{\mathfrak{g}, \mathbb{Q}}(\lambda)=\mathbb{Q}$ (see Lemma 3.4(4)). However, this contradicts Proposition 3.2(1), since $\lambda$ is not $\operatorname{Gal}(\overline{\mathbb{Q}} / \mathbb{Q})$-invariant.

In the remainder of this section, we consider each possible type of absolutely simple Lie algebra over $\mathbb{Q}$. The classical types are handled by using the calculations of $D_{\mathfrak{g}, F}(\lambda)$ in $[4$, $\S 27 \mathrm{~B}$, pp. 378-379], and the answers for ${ }^{1,2} E_{6}$ and $E_{7}$ follow from observations of Tits (see Section 3.5). The remaining types are very easy to deal with:

Lemma 3.7. Every absolutely simple Lie algebra of type $E_{8}, F_{4}$, or $G_{2}$ over $\mathbb{Q}$ is $\mathbb{R}$-universal.

Proof. These types have no outer automorphisms, so the $*$-action must be trivial. Furthermore, simple groups of these types have trivial center. Therefore, it is immediate from Remark 3.5 that $D_{\mathfrak{g}, \mathbb{Q}}(\lambda)=\mathbb{Q}$ for every dominant weight $\lambda$. So Lemma $3.4(3)$ implies that $\mathfrak{g}$ is $\mathbb{R}$-universal.

Erratum 3.8. We take this opportunity to correct the statements of Propositions 7.2 and 7.3(a) of [6]. The correct statement of Proposition 7.2 is:

Suppose $\mathfrak{g}_{\mathbb{R}}$ is a compact, simple Lie algebra over $\mathbb{R}$. There is a $\mathbb{Q}$-form $\mathfrak{g}_{\mathbb{Q}}$ of $\mathfrak{g}_{\mathbb{R}}$, such that $\mathfrak{g}_{\mathbb{Q}}$ splits over some quadratic extension of $\mathbb{Q}$, but is not $\mathbb{R}$-universal, if and only if either

(a) $\mathfrak{g}_{\mathbb{R}} \cong \mathfrak{s u}(n)$, for some $n$ that is divisible by 4 , or

(b) $\mathfrak{g}_{\mathbb{R}} \cong \mathfrak{s o}(n)$, for some $n \not \equiv 3,5(\bmod 8)($ with $n \geq 6)$.

The mistake originates in Proposition 7.3(a) of [6], where $\ell$ is required to only be odd, whereas it actually needs to be $\equiv 3(\bmod 4)$. This means that $\mathfrak{g}$, the compact real form of type $A_{\ell}$, is isomorphic to $\mathfrak{s u}(n)$, for some $n$ that is divisible by 4 . In Proposition 7.2 of [6], it was incorrectly stated that $n$ only needs to be even, not divisible by 4 .

\section{1 $\mathbb{R}$-universal Lie algebras of type $A$}

Proposition 3.9. Let $\mathfrak{g}=\mathfrak{s l}_{n}(D)$, where $D$ is a central division algebra over $\mathbb{Q}$. Then $\mathfrak{g}$ is $\mathbb{R}$-universal if and only if $D$ is either $\mathbb{Q}$ or a quaternion algebra that does not split over $\mathbb{R}$.

Proof. Let $d$ be the degree of $D$ over $\mathbb{Q}$, and let $\lambda$ be the highest weight of the standard representation of $\mathfrak{s l}_{d n}(\mathbb{C})$, so $\left\{\lambda^{i}\right\}_{i=1}^{d n}$ is a set of representatives of $Z^{*}$. Then $D_{\mathfrak{g}, \mathbb{Q}}\left(\lambda^{i}\right)$ and $D_{\mathfrak{g}, \mathbb{R}}\left(\lambda^{i}\right)$ are Brauer equivalent to the $i$-fold tensor products $D^{\otimes i}$ and $D_{\mathfrak{g}, \mathbb{R}}(\lambda)^{\otimes i}$, respectively [4, p. 378].

If $\mathfrak{g}$ is $\mathbb{R}$-universal, then, by taking $i=1$ and noting that $D_{\mathfrak{g}, \mathbb{R}}(\lambda)$ is either $\mathbb{R}$ or the quaternion algebra $\mathbb{H}$, we see from Lemma 3.4(4) that $D$ must be either $\mathbb{Q}$ or a quaternion algebra that does not split over $\mathbb{R}$.

Conversely, suppose $D$ is either $\mathbb{Q}$ or a quaternion algebra that does not split over $\mathbb{R}$. In either case, $D^{2}$ is Brauer equivalent to $\mathbb{Q}$, so $D_{\mathfrak{g}, \mathbb{Q}}\left(\lambda^{i}\right) \otimes_{\mathbb{Q}} \mathbb{R}$ is $\mathbb{Q} \otimes_{\mathbb{Q}} \mathbb{R}=\mathbb{R}$ if $i$ is even, and it is $D \otimes_{\mathbb{Q}} \mathbb{R}=\mathbb{H}$ if $i$ is odd. This is a division algebra for every $i$, so we see from Lemma 3.4(3) that $\mathfrak{g}$ is $\mathbb{R}$-universal. 
Proposition 3.10. Let $\mathfrak{g}=\mathfrak{s u}_{n}(B ; D, \tau)$, where

- $D$ is a division algebra that is central over a quadratic extension $L$ of $\mathbb{Q}$,

- $\tau$ is an anti-involution of $D$ that is nontrivial on $L$, and

- $B$ is an invertible $\tau$-Hermitian matrix in $\operatorname{Mat}_{n \times n}(D)$.

Then $\mathfrak{g}$ is $\mathbb{R}$-universal if and only if $D=L$ is an imaginary quadratic extension of $\mathbb{Q}$, and either $n$ is odd or $(-1)^{n / 2} \operatorname{det} B$ is either negative or the norm of some element of $L$.

Proof. We prove only $(\Rightarrow)$, but the argument is reversible. Since $\mathfrak{g}$ is outer, Lemma 3.6 implies that $\mathfrak{g} \otimes_{\mathbb{Q}} \mathbb{R}$ is also outer, and the quadratic extension $L$ is imaginary.

Let $d$ be the degree of $D$ over $L$, and let $\lambda$ be the highest weight of the standard representation of $\mathfrak{s l}_{d n}(\mathbb{C})$, so $\left\{\lambda^{i}\right\}_{i=1}^{d n}$ is a set of representatives of $Z^{*}$.

The Tits algebra of the natural representation $\rho: \mathfrak{g} \hookrightarrow \operatorname{Mat}_{n \times n}(D)$ is $D[4$, p. 378]. Since $D$ splits over $\mathbb{R}$ (recall that the center $L$ is an imaginary extension), we have $D_{\mathfrak{g}, \mathbb{R}}(\lambda)=\mathbb{C}$, so we conclude from Lemma 3.4(4) that $D=L$.

If $\lambda$ is any weight that is not fixed by the $*$-action, then $D_{\mathfrak{g}, \mathbb{Q}}(\lambda)=L$ and $D_{\mathfrak{g}, \mathbb{R}}(\lambda)=\mathbb{C}[4$, p. 378], so $D_{\mathfrak{g}, \mathbb{Q}}(\lambda) \otimes_{\mathbb{Q}} \mathbb{R}=D_{\mathfrak{g}, \mathbb{R}}(\lambda)$, as specified in Lemma 3.4(4). Hence, such weights (which are all of the weights when $n$ is odd) do not place any further restriction on $\mathfrak{g}$.

Suppose $n$ is even. Any weight that is fixed by the $*$-action (and is not in the root lattice) is congruent to $\lambda^{n / 2}$, modulo the root lattice. Write $L=\mathbb{Q}[\sqrt{a}]$ and let $b=(-1)^{n / 2} \operatorname{det} B$. Then, for $F$ either $\mathbb{Q}$ or $\mathbb{R}, D_{\mathfrak{g}, F}\left(\lambda^{n / 2}\right)$ is Brauer equivalent to the quaternion algebra $(a, b)_{F}[4$, p. 378 and Corollary 10.35 on p. 131)]. This is trivial in the Brauer group if and only if it is split, which means that $b$ is the norm of some element of $F[\sqrt{a}]$.

Since $\mathfrak{g}$ is $\mathbb{R}$-universal, we see from Lemma 3.4(4) that either $D_{\mathfrak{g}, \mathbb{R}}\left(\lambda^{n / 2}\right)=\mathbb{H}$ (which, by the preceding paragraph, means that $b$ is not a norm in $\mathbb{R}[\sqrt{a}]=\mathbb{C}$, so $b<0)$ or $D_{\mathfrak{g}, \mathbb{Q}}\left(\lambda^{n / 2}\right)=\mathbb{Q}$ (which means that $b$ is a norm in $\mathbb{Q}[\sqrt{a}]=L$ ).

\section{$3.2 \quad \mathbb{R}$-universal Lie algebras of type $B$}

Notation 3.11. Let $F \in\{\mathbb{Q}, \mathbb{R}\}$. Any symmetric matrix $B \in \mathrm{GL}_{k}(F)$ determines a nondegenerate quadratic form on $F^{k}$. We use $\operatorname{Cliff}_{F}^{0}(B)$ to denote the corresponding even Clifford algebra [5, p. 104].

It is well known that $\operatorname{Cliff}_{F}^{0}(B)$ is either a simple algebra or the direct sum of two isomorphic simple algebras over $F$ [5, Theorems 2.4 and 2.5, p. 110]. If $B$ has been diagonalized, then it is straightforward to determine whether this simple algebra is split (in which case, we also say that $\operatorname{Cliff}_{F}^{0}(B)$ is split). Namely, the simple algebra is Brauer equivalent to a quaternion algebra that can be calculated from the eigenvalues of $B$ (cf. [5, Corollary 3.14, p. 117]).

Example 3.12 ([5, pp. 122-125, and Corollary 2.10, p. 112]). Assume $B$ is a symmetric matrix in $\mathrm{GL}_{k}(\mathbb{R})$ with exactly $p$ positive eigenvalues (including multiplicity, so $k-p$ is the number of negative eigenvalues). Then $\operatorname{Cliff}_{\mathbb{R}}^{0}(B)$ is not split if and only if $2 p-k$ is congruent to 3 , 4 , or 5 , modulo 8 .

Proposition 3.13. Let $\mathfrak{g}=\mathfrak{s o}_{n}(B ; \mathbb{Q})$, where $B$ is a symmetric matrix in $\mathrm{GL}_{n}(\mathbb{Q})$, and $n$ is odd. Then $\mathfrak{g}$ is $\mathbb{R}$-universal if and only if either $\operatorname{Cliff}_{\mathbb{Q}}^{0}(B)$ is split or $\operatorname{Cliff}_{\mathbb{R}}^{0}(B)$ is not split.

Proof. Since the center of $\mathbf{S p i n}_{n}$ has order 2 when $n$ is odd, there is only one Tits algebra to consider (see Remark 3.5), and it is Brauer equivalent to $\operatorname{Cliff}_{F}^{0}(B)[4$, p. 378]. 


\section{$3.3 \quad \mathbb{R}$-universal Lie algebras of type $C$}

Example 3.14. For any $n$, the Lie algebra $\mathfrak{s p}_{2 n}(\mathbb{Q})$ is $\mathbb{R}$-universal. (More generally, every $\mathbb{Q}$ split semisimple Lie algebra is $\mathbb{R}$-universal, because it is clear from Definition 2.1 that $\beta_{\mathfrak{g}, F}(\lambda)$ is always trivial for $F$-split Lie algebras.)

Proposition 3.15. Let $\mathfrak{g}=\mathfrak{s u} \mathfrak{u}_{n}(B ; D, \tau)$, where

- $D$ is a quaternion division algebra over $\mathbb{Q}$,

- $\tau$ is the reversion anti-involution of $D$, and

- $B$ is an invertible $\tau$-Hermitian matrix in $\operatorname{Mat}_{n \times n}(D)$.

Then $\mathfrak{g}$ is $\mathbb{R}$-universal if and only if $D$ does not split over $\mathbb{R}$.

Proof. Since the center of $\mathbf{S} \mathbf{p}_{n}$ has order 2, there is only one Tits algebra to consider (see Remark 3.5). This Tits algebra is $D$ (over $\mathbb{Q}$ ) [4, p. 378], so the desired conclusion is immediate from Lemma 3.4(3).

\section{4 $\mathbb{R}$-universal Lie algebras of type $D$}

Lemma 3.6 implies that triality forms are not $\mathbb{R}$-universal. Therefore, all absolutely simple $\mathbb{R}$-universal Lie algebras of type $D$ are described in either Proposition 3.16 or Lemma 3.18.

Proposition 3.16. Let $\mathfrak{g}=\mathfrak{s o}_{2 k}(B ; \mathbb{Q})$, with $k \geq 3$, for some symmetric $B \in \mathrm{GL}_{2 k}(\mathbb{Q})$. Then $\mathfrak{g}$ is $\mathbb{R}$-universal if and only if either

(1) $\operatorname{Cliff}_{\mathbb{Q}}^{0}(B)$ is split, and $(-1)^{k} \operatorname{det} B$ is either negative or a square in $\mathbb{Q}$, or

(2) $\operatorname{Cliff}_{\mathbb{Q}}^{0}(B)$ does not split over $\mathbb{R}$, and $(-1)^{k} \operatorname{det} B$ is a square in $\mathbb{Q}$.

Proof. As in $\left[4, \S 27 \mathrm{~B}\right.$, type $D_{n}$, p. 379], let $\lambda, \lambda_{+}, \lambda_{-}$be dominant weights that represent the three nonzero elements of $Z^{*}$, with $\lambda$ being the highest weight of the natural representation of $\mathfrak{g}$ on $\mathbb{C}^{2 k}$. Then $D_{\mathfrak{g}, \mathbb{Q}}(\lambda)=\mathbb{Q}$ is trivial $[4$, p. 379].

Suppose, first, that $\mathfrak{g}$ is an inner form, which means that $(-1)^{k} \operatorname{det} B$ is a square in $\mathbb{Q}$. Then $\operatorname{Cliff}_{\mathbb{Q}}^{0}(B)$ is a direct sum of two algebras $C^{+}$and $C^{-}$that are Brauer equivalent to the full Clifford algebra [5, Theorem 2.5(3), p. 110] (and are therefore Brauer equivalent to a quaternion algebra). Furthermore, $D_{\mathfrak{g}, \mathbb{Q}}\left(\lambda_{ \pm}\right)$is Brauer equivalent to $C^{ \pm}[4$, p. 379]. Therefore, Lemma 3.4(3) shows that an inner form $\mathfrak{g}$ is:

- automatically $\mathbb{R}$-universal, when $\operatorname{Cliff}_{\mathbb{Q}}^{0}(B)$ does not split over $\mathbb{R}$, but

- $\mathbb{R}$-universal if and only if $\operatorname{Cliff}_{\mathbb{Q}}^{0}(B)$ is split, when $\operatorname{Cliff}_{\mathbb{Q}}^{0}(B)$ splits over $\mathbb{R}$.

Assume, now, that $\mathfrak{g}$ is an outer form. In order for $\mathfrak{g}$ to be $\mathbb{R}$-universal, $\mathfrak{g}$ must remain outer over $\mathbb{R}$ (see Lemma 3.6), which means $(-1)^{k} \operatorname{det} B<0$. Let $L$ be the (unique, imaginary) quadratic extension of $\mathbb{Q}$ over which $\mathfrak{g}$ becomes inner. Then $D_{\mathfrak{g}, \mathbb{Q}}\left(\lambda_{ \pm}\right)$is Brauer equivalent to $\operatorname{Cliff}_{\mathbb{Q}}^{0}(B)[4$, p. 379], which is central simple over $L$. Since $B$ splits over $\mathbb{R}$ (recall that the quadratic extension $L$ is imaginary), Lemma 3.4(3) implies that $\mathfrak{g}$ is $\mathbb{R}$-universal if and only if $\operatorname{Cliff}_{\mathbb{Q}}^{0}(B)$ is split.

Notation 3.17. The notion of even Clifford algebra was extended to the situation of Lemma 3.18 below by N. Jacobson. (A construction can be found in [4, $\S 8$, pp. 91ff].) We will denote this algebra by $C_{D}^{0}\left(B, \tau_{r}\right)$.

Lemma 3.18. Let $\mathfrak{g}=\mathfrak{s u}_{k}\left(B ; D, \tau_{r}\right)$, where 
- $D$ is a quaternion division algebra over $\mathbb{Q}$,

- $\tau_{r}$ is the reversion anti-involution, and

- $B$ is a $\tau_{r}$-Hermitian matrix in $\mathrm{GL}_{k}(D)$.

Then $\mathfrak{g}$ is $\mathbb{R}$-universal if and only if $D$ does not split over $\mathbb{R}$, and either

(1) $k$ is even and the reduced norm of $B$ (calculated in the algebra $\operatorname{Mat}_{k \times k}(D)$ ) is a square in $\mathbb{Q}$, or

(2) $k$ is odd and $C_{D}^{0}\left(B, \tau_{r}\right)$ is split.

Proof. As in the proof of Proposition 3.16, let $\lambda, \lambda_{+}, \lambda_{-}$be dominant weights that represent the three nonzero elements of $Z^{*}$, with $\lambda$ being the highest weight of the natural representation of $\mathfrak{g}$ on $(D \otimes \mathbb{Q} \mathbb{C})^{k}$.

$(\Rightarrow)$ We have $D_{\mathfrak{g}, \mathbb{Q}}(\lambda)=D[4$, p. 379], so $D$ does not split over $\mathbb{R}$ (see Lemma $3.4(3)$ ). (Note that this implies $\mathfrak{g} \otimes_{\mathbb{Q}} \mathbb{R} \cong \mathfrak{s o}_{k}(\mathbb{H})$.)

Suppose $k$ is even, so $\mathfrak{g} \otimes_{\mathbb{Q}} \mathbb{R}$ is inner. Then $\mathfrak{g}$ must also be inner (see Lemma 3.6). Since $k$ is even, this means that the reduced norm of $B$ is a square in $\mathbb{Q}$.

Suppose $k$ is odd. Then $\mathfrak{g} \otimes_{\mathbb{Q}} \mathbb{R}$ is outer, so the weights $\lambda_{+}$and $\lambda_{-}$are not fixed by the *-action. Therefore $D_{\mathfrak{g}, \mathbb{R}}\left(\lambda_{ \pm}\right)=\mathbb{C}$ (see Proposition 3.2). Since $D_{\mathfrak{g}, \mathbb{Q}}\left(\lambda_{ \pm}\right)$is Brauer equivalent to $C_{D}^{0}\left(B, \tau_{r}\right)[4$, p. 379], we conclude that $\mathfrak{g}$ is $\mathbb{R}$-universal if and only if this algebra is split (see Lemma 3.4(4)).

$(\Leftarrow)$ The proof for odd $k$ is reversible, so let us assume $k$ is even. Then $D_{\mathfrak{g}, \mathbb{R}}\left(\lambda_{ \pm}\right)=\mathbb{H}$ is nontrivial. (For example, $D_{\mathfrak{g}, \mathbb{R}}\left(\lambda_{ \pm}\right)$can be calculated by using $[11, \S 5.5]$.) Therefore $D_{\mathfrak{g}, \mathbb{Q}}\left(\lambda_{ \pm}\right)$ does not split over $\mathbb{R}$. Since $D_{\mathfrak{g}, \mathbb{Q}}\left(\lambda_{ \pm}\right)$is a quaternion algebra (because the center of $\mathbf{S p i n}_{2 k}$ has exponent 2 when $k$ is even), we conclude that $D_{\mathfrak{g}, \mathbb{Q}}\left(\lambda_{ \pm}\right) \otimes_{\mathbb{Q}} \mathbb{R} \cong D_{\mathfrak{g}, \mathbb{R}}\left(\lambda_{ \pm}\right)$, as desired.

\section{$3.5 \mathbb{R}$-universal Lie algebras of type $E_{6}$ and $E_{7}$}

It was pointed out in Lemma 3.7 that every absolutely simple Lie algebra of type $E_{8}, F_{4}$, or $G_{2}$ is $\mathbb{R}$-universal. In addition, the classical types were discussed in Sections 3.1-3.4. Therefore, the only types that remain are $E_{6}$ and $E_{7}$.

Definition 3.19 ([12, p. 649]). In the notation of Definition 2.1 , a Lie algebra $\mathfrak{g}$ over a field $F$ is strongly inner if $\mathbf{G}^{q}$ is split and the cohomology class $[\xi] \in H^{1}\left(F ; \overline{\mathbf{G}}^{q}\right)$ is the image of a cohomology class in $H^{1}\left(F ; \mathbf{G}^{q}\right)$ (under the map induced by the natural homomorphism $\mathbf{G}^{q} \rightarrow \overline{\mathbf{G}}^{q}$ ). This condition on $[\xi]$ is equivalent to requiring that $\delta_{*}[\xi]=0$ in $H^{2}\left(F ; Z\left(\mathbf{G}^{q}\right)\right)$, or, in other words, that $D_{\mathfrak{g}, F}(\lambda)=F$ for every dominant weight $\lambda$.

Proposition 3.20. Let $\mathfrak{g}$ be an absolutely simple Lie algebra over $\mathbb{Q}$ of type $E_{6}$. Then $\mathfrak{g}$ is $\mathbb{R}$-universal if and only if either

(1) $\mathfrak{g}$ is strongly inner (that is, of type ${ }^{1} E_{6,2}^{28}$ or ${ }^{1} E_{6,6}^{0}$ ), or

(2) $\mathfrak{g}$ is an outer form that splits over an imaginary quadratic extension of $\mathbb{Q}$.

Proof. Assume, first, that $\mathfrak{g}$ is inner. Since the center of any simply connected, almost simple group of type $E_{6}$ is cyclic of prime order (namely, it is of order 3 ), we see that $\mathfrak{g}$ is $\mathbb{R}$-universal if and only if either $\mathfrak{g}$ is strongly inner (over $\mathbb{Q}$ ) or $\mathfrak{g} \otimes_{\mathbb{Q}} \mathbb{R}$ is not strongly inner. From $[11, \S 6.4 .5]$, we see that Lie algebras of type ${ }^{1} E_{6,2}^{28}$ or ${ }^{1} E_{6,6}^{0}$ are always strongly inner, and those of type ${ }^{1} E_{6,2}^{16}$ are never strongly inner. In addition, a Lie algebra of type ${ }^{1} E_{6,0}^{78}$ over $\mathbb{Q}$ cannot be strongly inner [12, Propositions 4 and 5]. Since $\mathfrak{g} \otimes_{\mathbb{Q}} \mathbb{R}$ must be of type ${ }^{1} E_{6,2}^{28}$ or ${ }^{1} E_{6,6}^{0}$, it is strongly inner. Hence, $\mathfrak{g}$ is $\mathbb{R}$-universal if and only if it is strongly inner. 
Suppose, now, that $\mathfrak{g}$ is outer, and let $L$ be the unique quadratic extension of $\mathbb{Q}$ over which $\mathfrak{g}$ is inner. From Lemma 3.6, we know that if $\mathfrak{g}$ is $\mathbb{R}$-universal, then it must remain outer over $\mathbb{R}$, so $L$ is an imaginary extension of $\mathbb{Q}$. Let $\lambda$ be a weight that represents a nontrivial element of $Z^{*}$. Then $\lambda$ is not fixed by the $*$-action, so $D_{\mathfrak{g}, \mathbb{R}}(\lambda)=D_{\mathfrak{g}, \mathbb{C}}(\lambda)=\mathbb{C}$ and $D_{\mathfrak{g}, \mathbb{Q}}(\lambda)=D_{\mathfrak{g}, L}(\lambda)$ (see Proposition 3.2). So $\mathfrak{g}$ is $\mathbb{R}$-universal if and only if $\mathfrak{g} \otimes_{\mathbb{Q}} L$ is strongly inner.

Since $L$ is an imaginary extension, we know that $\mathfrak{g}$ splits at the infinite place of $L$. Then, by inspection of the possible Tits indices of type ${ }^{1} E_{6}$ over a nonarchimedean local field [10, p. 58], we see that the central vertex of the Tits index is circled at every place, so it must be circled over $L$ [2, Satz 4.3.3]. Therefore, $\mathfrak{g}$ must be either split or of type ${ }^{1} E_{6,2}^{16}$ over $L$. From [11, $\S 6.4 .5]$, we see that the form of type ${ }^{1} E_{6,2}^{16}$ is not strongly inner. Therefore, $\mathfrak{g}$ is $\mathbb{R}$-universal if and only if $\mathfrak{g} \otimes_{\mathbb{Q}} L$ is split.

Proposition 3.21. Let $\mathfrak{g}$ be an absolutely simple Lie algebra over $\mathbb{Q}$ of type $E_{7}$. Then $\mathfrak{g}$ is $\mathbb{R}$-universal if and only if either

(1) $\mathfrak{g}$ is of type $E_{7,3}^{28}$ or $E_{7,7}^{0}($ over $\mathbb{Q})$, or

(2) $\mathfrak{g} \otimes_{\mathbb{Q}} \mathbb{R}$ is of type $E_{7,0}^{133}, E_{7,2}^{31}$, or $E_{7,4}^{9}$.

Proof. Since a simply connected group of type $E_{7}$ has a center of order 2 , there is only one Tits algebra to consider. For $\mathfrak{g}$ to be $\mathbb{R}$-universal, this algebra needs to either be trivial over $\mathbb{Q}$ or nontrivial over $\mathbb{R}$. Tits $[11, \S 6.5 .5]$ points out that it is trivial (over any field) for types $E_{7,3}^{28}$ and $E_{7,7}^{0}$, but nontrivial (over any field) for types $E_{7,2}^{31}$ and $E_{7,4}^{9}$. Since there are no strongly inner anisotropic groups of type $E_{7}$ over $\mathbb{Q}$ or $\mathbb{R}$ [12, Propositions 4 and 5], the Tits algebra is also nontrivial for the anisotropic Lie algebra $E_{7,0}^{133}$ (over the fields of interest to us).

\section{$4 \mathbb{R}$-universal Lie algebras that are not absolutely simple}

Section 3 lists the $\mathbb{R}$-universal Lie algebras that are absolutely simple. It is easy to describe the rest of the simple ones:

Proposition 4.1. Let $\mathfrak{g}$ be a simple Lie algebra over $\mathbb{Q}$ that is not absolutely simple. Then $\mathfrak{g}$ is $\mathbb{R}$-universal if and only if $\mathfrak{g}=\mathbf{R}_{L / \mathbb{Q}} \mathfrak{g}^{\prime}$ is the restriction of scalars of a strongly inner, absolutely simple Lie algebra $\mathfrak{g}^{\prime}$ over an imaginary quadratic extension $L$ of $\mathbb{Q}$.

Proof. $(\Rightarrow)$ We have $\mathfrak{g}=\mathbf{R}_{L / \mathbb{Q}} \mathfrak{g}^{\prime}$, for some absolutely simple Lie algebra $\mathfrak{g}^{\prime}$ over some finite extension $L$ of $\mathbb{Q}[10, \S 3.1 .2]$. From Lemma 3.6, we see that if $\mathfrak{g}$ is $\mathbb{R}$-universal, then the extension $L$ must be imaginary quadratic.

Let $\lambda^{\prime}$ be a nonzero dominant weight of $\mathfrak{g}^{\prime}$, and let $\lambda=\mathbf{R}_{L / \mathbb{Q}} \lambda^{\prime}$ be the corresponding weight of $\mathfrak{g}$. Then $\lambda$ is not fixed by the $*$-action, so we have $D_{\mathfrak{g}, F}(\lambda)=D_{\mathfrak{g}, L}(\lambda)=D_{\mathfrak{g}^{\prime}, L}(\lambda)$. (The final equality is because $\mathfrak{g}=\mathbf{R}_{L / \mathbb{Q}} \mathfrak{g}^{\prime}$.) On the other hand, since all Lie algebras are split over $\mathbb{C}$, we have $D_{\mathfrak{g}, \mathbb{R}}(\lambda)=D_{\mathfrak{g}, \mathbb{C}}(\lambda)=\mathbb{C}$. Hence, we see from Lemma 3.4(4) that if $\mathfrak{g}$ is $\mathbb{R}$-universal, then $D_{\mathfrak{g}^{\prime}, L}(\lambda)=L$. Since this is true for every dominant weight $\lambda$ of $\mathfrak{g}^{\prime}$, we conclude that $\mathfrak{g}^{\prime}$ is strongly inner.

$(\Leftarrow)$ Note that, since $\mathfrak{g}^{\prime}$ is strongly inner, the simply connected group $\mathbf{G}^{\prime}$ corresponding to $\mathfrak{g}^{\prime}$ may be written as $\mathbf{G}^{\prime}={ }^{\zeta} \mathbf{G}^{s}$, where $\mathbf{G}^{s}$ is split (and simply connected) and $\zeta \in H^{1}\left(L ; \mathbf{G}^{s}\right)$. By Restriction of Scalars, then $\mathbf{G}={ }^{\xi} \mathbf{G}^{q}$, where $\mathbf{G}^{q}=\mathbf{R}_{L / \mathbb{Q}} \mathbf{G}^{s}$ is quasi-split and $\xi \in H^{1}\left(\mathbb{Q} ; \mathbf{G}^{q}\right)$. Then, in the notation of Definition 2.1, we have $\delta_{*}[\xi]=0$, so $\beta_{\mathfrak{g}, F}(\lambda)=0$ for every $*$-invariant weight $\lambda$. It is therefore easy to see that $\mathfrak{g}$ is $\mathbb{R}$-universal.

We also briefly describe the considerations involved in constructing semisimple $\mathbb{R}$-universal Lie algebras from simple ones: 
Proposition 4.2. A direct sum $\mathfrak{g}=\mathfrak{g}_{1} \oplus \cdots \oplus \mathfrak{g}_{r}$ of simple Lie algebras over $\mathbb{Q}$ is $\mathbb{R}$-universal if and only if:

(1) each $\mathfrak{g}_{i}$ is $\mathbb{R}$-universal,

(2) either $\mathfrak{g}$ is inner, or it becomes inner over an imaginary quadratic extension $L$ of $\mathbb{Q}$, and the $*$-action of $\mathrm{Gal}(\mathbb{C} / \mathbb{R})$ is the same as the $*$-action of $\operatorname{Gal}(L / \mathbb{Q})$.

(3) we have $D_{\mathfrak{g}_{i}, \mathbb{Q}}\left(\lambda_{i}\right) \cong D_{\mathfrak{g}_{j}, \mathbb{Q}}\left(\lambda_{j}\right)$ whenever $\lambda_{i}$ and $\lambda_{j}$ are $*$-invariant dominant weights of $G_{i}$ and $G_{j}$, such that $D_{\mathfrak{g}_{i}, \mathbb{Q}}\left(\lambda_{i}\right) \neq \mathbb{Q}$ and $D_{\mathfrak{g}_{j}, \mathbb{Q}}\left(\lambda_{j}\right) \neq \mathbb{Q}$, and

(4) either $\mathfrak{g}$ is inner, or it becomes inner over an imaginary quadratic extension $L$ of $\mathbb{Q}$, such that $D_{\mathfrak{g}_{i}, \mathbb{Q}}\left(\lambda_{i}\right)$ splits over $L$, for every dominant weight $\lambda_{i}$ of every $\mathfrak{g}_{i}$ that is inner $($ over $\mathbb{Q})$.

Proof. We prove only $(\Rightarrow)$, but the argument is reversible. (We use $\sim$ to denote "is Brauer equivalent to".)

(1) Any representation $\rho_{i}$ of $\mathfrak{g}_{i}$ extends to a representation of $\mathfrak{g}$ (that is 0 on the other simple factors), so it is clear that $\mathfrak{g}_{i}$ must be $\mathbb{R}$-universal.

(2) See Lemma 3.6. (This means that all $\mathfrak{g}_{i}$ become inner over the same quadratic extension L.)

(3) We have

$$
\begin{aligned}
D_{\mathfrak{g}, \mathbb{R}}\left(\lambda_{i}+\lambda_{j}\right) & \sim D_{\mathfrak{g}, \mathbb{R}}\left(\lambda_{i}\right) \otimes_{\mathbb{R}} D_{\mathfrak{g}, \mathbb{R}}\left(\lambda_{j}\right) \\
& \cong\left(D_{\mathfrak{g}, \mathbb{Q}}\left(\lambda_{i}\right) \otimes_{\mathbb{Q}} \mathbb{R}\right) \otimes_{\mathbb{R}}\left(D_{\mathfrak{g}, \mathbb{Q}}\left(\lambda_{i}\right) \otimes_{\mathbb{Q}} \mathbb{R}\right) \cong \mathbb{H} \otimes_{\mathbb{R}} \mathbb{H} \sim \mathbb{R} .
\end{aligned}
$$

Since $\mathfrak{g}$ is $\mathbb{R}$-universal, this implies $D_{\mathfrak{g}, \mathbb{Q}}\left(\lambda_{i}+\lambda_{j}\right) \sim \mathbb{Q}$, from which we conclude that $D_{\mathfrak{g}, \mathbb{Q}}\left(\lambda_{i}\right) \otimes_{\mathbb{Q}}$ $D_{\mathfrak{g}, \mathbb{Q}}\left(\lambda_{j}\right) \sim \mathbb{Q}$, so $D_{\mathfrak{g}, \mathbb{Q}}\left(\lambda_{i}\right) \cong D_{\mathfrak{g}, \mathbb{Q}}\left(\lambda_{j}\right)$ (since these are quaternion algebras).

(4) Let $\lambda_{j}$ be a dominant weight of some (outer) simple factor $G_{j}$ that is not fixed by the $*$-action. Then $\lambda_{i}+\lambda_{j}$ is not fixed by the $*$-action, so

$$
D_{\mathfrak{g}, \mathbb{Q}}\left(\lambda_{i}+\lambda_{j}\right)=D_{\mathfrak{g}, L}\left(\lambda_{i}+\lambda_{j}\right) \sim D_{\mathfrak{g}_{i}, L}\left(\lambda_{i}\right) \otimes_{L} D_{\mathfrak{g}_{j}, L}\left(\lambda_{j}\right)=D_{\mathfrak{g}_{i}, L}\left(\lambda_{i}\right) \otimes_{L} L \cong D_{\mathfrak{g}_{i}, L}\left(\lambda_{i}\right) .
$$

Since $D_{\mathfrak{g}, \mathbb{R}}\left(\lambda_{i}+\lambda_{j}\right)=D_{\mathfrak{g}, \mathbb{C}}\left(\lambda_{i}+\lambda_{j}\right)=\mathbb{C}$, and $\mathfrak{g}$ is $\mathbb{R}$-universal, we conclude from Lemma 3.4(4) that $D_{\mathfrak{g} i, L}\left(\lambda_{i}\right)=L$. Since $D_{\mathfrak{g}, \mathbb{Q}}\left(\lambda_{i}\right) \otimes_{\mathbb{Q}} L \sim D_{\mathfrak{g}, L}\left(\lambda_{i}\right)$, this means that $D_{\mathfrak{g}, \mathbb{Q}}\left(\lambda_{i}\right)$ splits over $L$.

\section{Acknowledgements}

It is a pleasure to thank V. Chernousov for a very helpful discussion about Tits algebras of special orthogonal groups, A. Rapinchuk for explaining how to prove Lemma 2.5, and the anonymous referees for numerous very insightful comments on a previous version of this manuscript, including some important corrections.

\section{References}

[1] Demazure M., Grothendieck A., Schémas en groups. III. Structure des schémas en groupes reductifs, Séminaire de géométrie algébrique du Bois Marie, 1962/1964, available at http://library.msri.org/ books/sga/sga/pdf/sga3-3.pdf.

[2] Harder G., Bericht über neuere Resultate der Galoiskohomologie halbeinfacher Gruppen, Jber. Deutsch. Math.-Verein. 70 (1968), 182-216, available at http://www.digizeitschriften.de/en/dms/img/?PPN= PPN37721857X_0070\&DMDID=dmdlog17.

[3] Kneser M., Lectures on Galois cohomology of classical groups, Tata Institute of Fundamental Research Lectures on Mathematics, Vol. 47, Tata Institute of Fundamental Research, Bombay, 1969, available at http://www.math.tifr.res.in/ publ/ln/tifr47.pdf.

[4] Knus M.A., Merkurjev A., Rost M., Tignol J.P., The book of involutions, American Mathematical Society Colloquium Publications, Vol. 44, Amer. Math. Soc., Providence, RI, 1998. 
[5] Lam T.Y., Introduction to quadratic forms over fields, Graduate Studies in Mathematics, Vol. 67, Amer. Math. Soc., Providence, RI, 2005.

[6] Morris D., Real representations of semisimple Lie algebras have $\mathbb{Q}$-forms, in Algebraic Groups and Arithmetic, Tata Institute of Fundamental Research, Mumbai, 2004, 469-490.

[7] Platonov V., Rapinchuk A., Algebraic groups and number theory, Pure and Applied Mathematics, Vol. 139, Academic Press, Inc., Boston, MA, 1994.

[8] Prasad G., Rapinchuk A., On the existence of isotropic forms of semi-simple algebraic groups over number fields with prescribed local behavior, Adv. Math. 207 (2006), 646-660.

[9] Raghunathan M.S., Arithmetic lattices in semisimple groups, Proc. Indian Acad. Sci. Math. Sci. 91 (1982), $133-138$.

[10] Tits J., Classification of algebraic semisimple groups, in Algebraic Groups and Discontinuous Subgroups (Proc. Sympos. Pure Math., Boulder, Colo., 1965), Amer. Math. Soc., Providence, R.I., 1966, 33-62.

[11] Tits J., Représentations linéaires irréductibles d'un groupe réductif sur un corps quelconque, J. Reine Angew. Math. 247 (1971), 196-220.

[12] Tits J., Strongly inner anisotropic forms of simple algebraic groups, J. Algebra 131 (1990), 648-677. 